\title{
ON A SINGULAR INTEGRAL EQUATION WITH LOG KERNEL AND ITS APPLICATION
}

\author{
SUDESHNA BANERJEA AND CHIRANJIB SARKAR
}

Received 11 January 2005; Revised 12 June 2006; Accepted 21 June 2006

We used function theoretic method to solve a singular integral equation with logarithmic kernel in two disjoint finite intervals where the unknown function satisfying the integral equation may be bounded or unbounded at the nonzero finite endpoints of the interval concerned. An appropriate solution of this integral equation is then applied to solve the problem of scattering of time harmonic surface water waves by a fully submerged thin vertical barrier with a single gap.

Copyright @ 2006 Hindawi Publishing Corporation. All rights reserved.

\section{Introduction}

The following singular integral equation with logarithmic kernel arises in various scattering and radiation problems of linear water wave theory involving vertical barriers:

$$
\int_{G} g(u) \ln \left|\frac{x+u}{x-u}\right| d u=f(x), \quad x \in G
$$

where $g$ and $f$ are differentiable functions and $G$ may consist of single or disjoint multiple intervals.

Usually in the literature, (1.1) was solved by reducing it to a Cauchy-type singular integral equation and consequently this solution was utilized to solve the corresponding water wave problem. The solution of (1.1) obtained by reducing it to Cauchy-type singular integral equation posed difficulty in solving the corresponding scattering or radiation problem due to the fact that the weak singularity in (1.1) was converted to strong singularity of Cauchy type. This difficulty was taken care of by Chakrabarti et al. [3], Manam [5], while solving the problem of scattering of water waves by a vertical wall with a gap. They used function theoretic method to solve the corresponding integral equation (1.1) with $G \equiv(a, b)$ or $G \equiv(0, a) \cup(b, c)$ directly instead of reducing it to Cauchy-type singular integral equation. In the present paper, we have used function-theoretic method to 
2 Weakly singular integral equation

solve (1.1) with $G \equiv(0, a) \cup(b, c)$ under the condition when

(i)

$$
g(u) \sim O\left(|(u-p)|^{-1 / 2}\right), \quad \text { as } u \longrightarrow p,
$$

(ii)

$$
g(u) \sim O\left(|(u-p)|^{1 / 2}\right), \quad \text { as } u \longrightarrow p
$$

$p$ being $a, b$ or $c$. Utilizing the boundedness of $g(u)$ at $a, b, c$ given by (1.3), the problem of scattering of water waves by a submerged vertical wall with a gap is solved completely. This problem was solved earlier by Chakrabarti and Vijaya Bharathi [4] and Banerjea and Mandal [2]. In the present paper, we have used a very simple method to solve the problem completely. In Section 2, we have obtained the solution of a singular integral equation (1.1) with $G \equiv(0, a) \cup(b, c)$ where $g(u)$ satisfies (1.2) and (1.3). In Section 3, we have formulated the problem of scattering of water waves by a vertical wall submerged in water and discussed the genesis of (1.1). Then we used the boundedness property of $g(u)$ given by (1.3) to obtain solution of the scattering problem in a simple manner. Chakrabarti and Vijaya Bharathi [4] used complex variable technique while Banerjea and Mandal [2] used two types of singular integral equation method to solve the problem. The singular integral equations arising in [2] consist of Cauchy kernel and a combination of logarithmic and Cauchy kernel. In both methods in [2], the weakly singular kernel was converted to strong singular kernel. In the present paper, we have reduced the corresponding boundary value problem to (1.1) with weakly singular kernel when $G \equiv(0, a) \cup(b, c)$.

\section{Method of solution of a singular integral equation with logarithmic kernel}

Let us consider the equation

$$
\frac{1}{\pi} \int_{G} g(t) \ln \left|\frac{y+t}{y-t}\right| d t=f(y), \quad y \in G, \quad G \equiv(0, a) \cup(b, c),
$$

where $f(y)$ is a known function and $g(u)$ is an unknown function which satisfies (1.2). Here $f(u)$ and $g(u)$ are both differentiable functions.

For solution of (2.1) under the condition (1.2), let

$$
F(z)=\frac{d}{d z} \int_{G} g(u) \ln \left(\frac{u+z}{u-z}\right) d u,
$$

where $F(z)$ is sectionally analytic function in complex $z$-plane cut along $(-c,-b) \cup$ $(-a, 0) \cup(0, a) \cup(b, c)$ and $F(z) \sim O\left(1 / z^{2}\right)$ as $|z| \rightarrow \infty$.

We denote

$$
F^{ \pm}(x)=\lim _{y \rightarrow 0 \pm} F(z)
$$


S. Banerjea and C. Sarkar 3

Using Plemelj's formula we have

$$
\begin{gathered}
F^{+}(x)+F^{-}(x)=\kappa(x), \\
F^{+}(x)-F^{-}(x)=\lambda(x), \\
\lambda(x)= \begin{cases}2 \pi f^{\prime}(x), & x \in G, \\
2 \pi f^{\prime}(-x), & x \in G^{\prime},\end{cases} \\
\lambda(x)= \begin{cases}2 \pi i g^{\prime}(x), & x \in G, \\
-2 \pi i g^{\prime}(-x), & x \in G^{\prime},\end{cases}
\end{gathered}
$$

and $G \equiv(0, a) \cup(b, c)$ and $G^{\prime} \equiv(-c,-b) \cup(-a, 0)$.

The first equation of (2.4) defines a Riemann-Hilbert problem for $F(z)$, the solution of which is given by

$$
F(z)=F_{0}(z)\left[D z^{2}+B z+C+\frac{1}{2 \pi i} \int_{G \cup G^{\prime}} \frac{\kappa(t)}{F_{0}^{+}(t)} \frac{d t}{t-z}\right],
$$

where $D, B, C$ are unknown constants and

$$
F_{0}(z)=\left\{\left(z^{2}-a^{2}\right)\left(z^{2}-b^{2}\right)\left(z^{2}-c^{2}\right)\right\}^{-1 / 2} .
$$

Using Plemelj's formula in (2.7) and utilizing (2.4), (2.5), and (2.6), we get the expression for the function $g$ as

$$
\begin{gathered}
g(x)=\frac{1}{\pi} \frac{1}{R(x)}\left[D x^{2}+B x+C+2 x P(x)\right], \quad \text { in } 0<x<a, \\
g(-x)=\frac{1}{\pi} \frac{1}{R(x)}\left[-D x^{2}-B x-C-2 x P(x)\right], \quad \text { in }-a<x<0,
\end{gathered}
$$

and also

$$
\begin{array}{r}
g(x)=\frac{1}{\pi} \frac{1}{-R(x)}\left[D x^{2}+B x+C+2 x P(x)\right], \quad \text { in } b<x<c, \\
g(-x)=\frac{1}{\pi} \frac{1}{-R(x)}\left[-D x^{2}-B x-C-2 x P(x)\right], \quad \text { in }-c<x<-b .
\end{array}
$$

Comparing (2.9) with (2.10) and (2.11) with (2.12), we get

$$
D=C=0 .
$$

Thus,

$$
g(u)= \begin{cases}\frac{2 u}{R(u)}\left[B_{0}+P(u)\right], & 0<u<a, \\ \frac{-2 u}{R(u)}\left[B_{0}+P(u)\right], & b<u<c,\end{cases}
$$


4 Weakly singular integral equation

where $B_{0}=B / 2$ is a constant to be determined

$$
\begin{gathered}
P(u)=\int_{G} \frac{f^{\prime}(t) S(t)}{u^{2}-t^{2}} d t, \\
R(x)=\left|\left\{\left(a^{2}-x^{2}\right)\left(b^{2}-x^{2}\right)\left(c^{2}-x^{2}\right)\right\}^{1 / 2}\right|, \\
S(u)= \begin{cases}R(x), & 0<x<a, \\
-R(x), & b<x<c .\end{cases}
\end{gathered}
$$

In order to determine $B_{0}$, we proceed as follows.

By direct integration, we get from (2.14),

$$
\int_{G} u g(u) d u=B_{0} \frac{1}{\pi} \int_{G} \frac{2 u^{2}}{S(u)} d u .
$$

Multiplying both sides of $(2.1)$ with $1 / S(x)$ and $x / S(x)$ and integrating over $G$, we get the following two relations:

$$
\begin{aligned}
& \int_{G} \frac{f(x)}{S(x)} d x=\frac{I_{1}(a)}{\pi} \int_{0}^{a} g(u) d u+\frac{I_{1}(c)}{\pi} \int_{b}^{c} g(u) d u, \\
& \int_{G} \frac{x f(x)}{S(x)} d x=\frac{I_{2}(a)}{\pi} \int_{0}^{a} g(u) d u+\frac{I_{2}(c)}{\pi} \int_{b}^{c} g(u) d u
\end{aligned}
$$

with

$$
\begin{aligned}
& I_{1}(u)=\int_{G} \frac{1}{S(x)} \ln \left|\frac{u+x}{u-x}\right| d x, \\
& I_{2}(u)=\int_{G} \frac{x}{S(x)} \ln \left|\frac{u+x}{u-x}\right| d x .
\end{aligned}
$$

Multiplying (2.1) by $T(x)$, where

$$
T(x)=\left\{\frac{x^{2}\left(x^{2}-b^{2}\right)}{\left(x^{2}-a^{2}\right)\left(c^{2}-x^{2}\right)}\right\}^{1 / 2}
$$

and integrating over $G$, and using the following result:

$$
\begin{aligned}
I_{3}(u) & =\int_{G} T(x) \ln \left|\frac{u+x}{u-x}\right| d x \\
& = \begin{cases}\pi(u-a)+I_{3}(a), & 0<u<a, \\
\pi(u-c)+I_{3}(c), & b<u<c,\end{cases}
\end{aligned}
$$

we get

$$
\begin{aligned}
\int_{G} u g(u) d u= & \frac{\left[a \pi-I_{3}(a)\right]}{\pi} \int_{0}^{a} g(u) d u+\frac{\left[c \pi-I_{3}(c)\right]}{\pi} \int_{b}^{c} g(u) d u \\
& +\int_{G} f(x) T(x) d x .
\end{aligned}
$$


Now, from (2.19), we get

$$
\begin{aligned}
& \int_{0}^{a} g(u) d u=\frac{\pi}{\triangle}\left[I_{2}(c) \int_{G} \frac{f(x)}{S(x)} d x-I_{1}(c) \int_{G} \frac{x f(x)}{S(x)} d x\right], \\
& \int_{b}^{c} g(u) d u=\frac{\pi}{\triangle}\left[I_{1}(a) \int_{G} \frac{x f(x)}{S(x)} d x-I_{2}(c) \int_{G} \frac{f(x)}{S(x)} d x\right],
\end{aligned}
$$

where

$$
\triangle=\left[I_{1}(a) I_{2}(c)-I_{1}(c) I_{2}(a)\right]
$$

Hence by (2.23), using (2.24) and (2.25), we get

$$
\int_{G} u g(u) d u=\frac{K_{1}}{\triangle} \int_{G} \frac{f(x)}{S(x)} d x+\frac{K_{2}}{\triangle} \int_{G} \frac{x f(x)}{S(x)} d x+\int_{G} f(x) T(x) d x,
$$

where

$$
\begin{aligned}
& K_{1}=\left[\pi\left\{a I_{2}(c)-c I_{2}(a)\right\}+\left\{I_{2}(a) I_{3}(c)-I_{2}(c) I_{3}(a)\right\}\right], \\
& K_{2}=\left[\pi\left\{c I_{1}(a)-a I_{1}(c)\right\}+\left\{I_{1}(c) I_{3}(a)-I_{1}(a) I_{3}(c)\right\}\right] .
\end{aligned}
$$

And finally, using (2.18) and (2.27), the unknown constant $B_{0}$ is determined.

Thus the solution of (1.1) with $g(u)$ satisfying (1.2) is given by (cf. (2.14)),

$$
g(u)= \begin{cases}\frac{1}{\pi} \frac{2 u}{R(u)}\left[B_{0}+P(u)\right], & 0<u<a, \\ -\frac{1}{\pi} \frac{2 u}{R(u)}\left[B_{0}+P(u)\right], & b<u<c,\end{cases}
$$

where $B_{0}$ is a known constant.

Making $a \rightarrow 0$ in (2.29), the results in [3] can be recovered.

Next, we find the solution of $(2.1)$ with $g(u)$ satisfying (1.3). We rewrite $P(x)$ in $(2.15)$ as follows:

$$
\begin{aligned}
P(x)= & \left(a^{2}-x^{2}\right)\left(b^{2}-x^{2}\right)\left(c^{2}-x^{2}\right) \int_{0}^{a} \frac{f^{\prime}(t)}{\left(x^{2}-t^{2}\right) R(t)} d t \\
& -\left(x^{2}-a^{2}\right)\left(x^{2}-b^{2}\right)\left(c^{2}-x^{2}\right) \int_{b}^{c} \frac{f^{\prime}(t)}{\left(x^{2}-t^{2}\right) R(t)} d t \\
& +\left(a^{2}-x^{2}\right)\left(b^{2}-x^{2}\right)\left[\int_{0}^{a} \frac{f^{\prime}(t)}{R(t)} d t-\int_{b}^{c} \frac{f^{\prime}(t)}{R(t)} d t\right] \\
& +\left(a^{2}-x^{2}\right)\left[\int_{0}^{a} \sqrt{\frac{\left(c^{2}-t^{2}\right)}{\left(a^{2}-t^{2}\right)\left(b^{2}-t^{2}\right)}} f^{\prime}(t) d t-\int_{b}^{c} \sqrt{\frac{\left(c^{2}-t^{2}\right)}{\left(t^{2}-a^{2}\right)\left(t^{2}-b^{2}\right)}} f^{\prime}(t) d t\right] \\
& +\left[\int_{0}^{a} \sqrt{\frac{\left(c^{2}-t^{2}\right)\left(b^{2}-t^{2}\right)}{\left(a^{2}-t^{2}\right)}} f^{\prime}(t) d t-\int_{b}^{c} \sqrt{\frac{\left(c^{2}-t^{2}\right)\left(t^{2}-b^{2}\right)}{\left(t^{2}-a^{2}\right)}} f^{\prime}(t) d t\right] .
\end{aligned}
$$


Substituting (2.30) into (2.29), we observe that for $g(u)$ to satisfy $(1.3)$, we must have the following relations:

$$
\begin{gathered}
{\left[\int_{0}^{a} \frac{\sqrt{\left(c^{2}-t^{2}\right)\left(b^{2}-t^{2}\right)} f^{\prime}(t)}{\left\{\left(a^{2}-t^{2}\right)\right\}^{1 / 2}} d t+\int_{b}^{c} \frac{\sqrt{\left(t^{2}-b^{2}\right)\left(c^{2}-t^{2}\right)} f^{\prime}(t)}{\left\{\left(t^{2}-a^{2}\right)\right\}^{1 / 2}} d t\right]+B_{0}=0,} \\
{\left[\int_{0}^{a} \frac{\sqrt{\left(c^{2}-t^{2}\right)} f^{\prime}(t)}{\left\{\left(a^{2}-t^{2}\right)\left(b^{2}-t^{2}\right)\right\}^{1 / 2}} d t-\int_{b}^{c} \frac{\sqrt{\left(c^{2}-t^{2}\right)} f^{\prime}(t)}{\left\{\left(t^{2}-a^{2}\right)\left(t^{2}-b^{2}\right)\right\}^{1 / 2}} d t\right]=0,} \\
{\left[\int_{0}^{a} \frac{f^{\prime}(t)}{R(t)} d t-\int_{b}^{c} \frac{f^{\prime}(t)}{R(t)} d t\right]=0 .}
\end{gathered}
$$

These are three sovability conditions which $f(t)$ must satisfy in order that the solution of (1.1) satisfying (1.3) exists.

Here the solution of (1.1) is given by

$$
g(x)= \begin{cases}\frac{2 x}{\pi} R(x)\left[\int_{0}^{a} \frac{f^{\prime}(t)}{\left(x^{2}-t^{2}\right) R(t)} d t-\int_{b}^{c} \frac{f^{\prime}(t)}{\left(x^{2}-t^{2}\right) R(t)} d t\right], \quad 0<x<a, \\ \frac{-2 x}{\pi} R(x)\left[\int_{0}^{a} \frac{f^{\prime}(t)}{\left(x^{2}-t^{2}\right) R(t)} d t-\int_{b}^{c} \frac{f^{\prime}(t)}{\left(x^{2}-t^{2}\right) R(t)} d t\right], \quad b<x<c,\end{cases}
$$

where $R(t)$ is given by (2.16).

In the next section, we will consider the problem of scattering of surface water waves by a submerged vertical barrier with a gap and reduce the corresponding boundary value problem to the integral equation (2.1).

\section{Genesis of the integral equation mathematical formulation of the scattering problem}

We consider the irrotational motion of an incompressible inviscid fluid under the action of gravity and use a rectangular Cartesian coordinate system in which the $y$-axis is taken vertically downwards, so that the fluid region occupies the region $y>0 ; x \in \mathbb{R}$, and the vertical barrier occupies the region $x=0 ; y \in \mathbf{B}$, where $\mathbf{B} \equiv[a, b] \cup[c, \infty)$. Assuming the harmonic time dependence $e^{-i \sigma t}(\sigma>0)$ in the velocity potential $\Phi(x, y, t)=$ $R\left\{\phi(x, y) e^{i \sigma t}\right\}$, the problem under consideration is that of solving the following boundary value problem for $\phi$ :

$$
\frac{\partial^{2} \phi}{\partial x^{2}}+\frac{\partial^{2} \phi}{\partial y^{2}}=0, \quad y>0, x \in \mathbb{R}
$$

with

$$
\frac{\partial \phi}{\partial y}+K \phi=0, \quad \text { on } y=0
$$


where $K=\sigma^{2} / \mathrm{g}$, g being the gravitational acceleration,

$$
\begin{gathered}
\frac{\partial \phi}{\partial x}=0, \quad \text { on } x=0 \pm, y \in \mathbf{B}, \\
\phi(0+, y)=\phi(0-, y), \quad y \in G(\text { gap }), \quad[G \equiv(0, a) \cup(b, c)], \\
r^{1 / 2} \nabla \phi \text { is bounded } \quad \text { as } r \longrightarrow 0,
\end{gathered}
$$

" $r$ " being the distance from sharp edges of the barrier,

$$
\phi \sim \begin{cases}(1-R) e^{-K y+i K x}, & x \longrightarrow \infty, \\ e^{-K y+i K x}+R e^{-K y-i K x}, & x \longrightarrow-\infty,\end{cases}
$$

where $R$ is the reflection coefficient.

3.1. Reduction of scattering problem to singular integral equation with logarithmic kernel. We first express $\phi(x, y)$ by using Havelock's expansion satisfying (3.1) and the conditions (3.2) and (3.6),

$$
\phi \sim \begin{cases}(1-R) e^{-K y+i K x}+\int_{0}^{\infty} A(\xi) L(\xi, y) e^{-\xi x} d \xi, & x>0, \\ e^{-K y+i K x}+R e^{-K y-i K x}-\int_{0}^{\infty} A(\xi) L(\xi, y) e^{-\xi x} d \xi, & x<0,\end{cases}
$$

where $L(\xi, y)=\xi \cos \xi y-K \sin \xi y$ and $A(\xi)$ is unknown.

For satisfying (3.3), (3.4), and (3.5), the unknown function $A(\xi)$ must satisfy the set of dual integral equation

$$
\begin{gathered}
\int_{0}^{\infty} A(\xi) L(\xi, y) d \xi=R e^{-K y}, \quad y \in G, \\
\int_{0}^{\infty} \xi A(\xi) L(\xi, y) d \xi=i K(1-R) e^{-K y}, \quad y \in \mathbf{B},
\end{gathered}
$$

which can be alternatively written as

$$
\begin{gathered}
\int_{0}^{\infty} A(\xi) \sin \xi y d \xi= \begin{cases}D_{1} e^{K y}-\frac{R}{2 K} e^{-K y}, & 0<y<a, \\
D_{2} e^{K y}-\frac{R}{2 K} e^{-K y}, & b<y<c,\end{cases} \\
\int_{0}^{\infty} \xi A(\xi) \sin \xi y d \xi= \begin{cases}E_{1} e^{K y}-\frac{i(1-R)}{2} e^{-K y}, & a<y<b, \\
E_{2} e^{K y}-\frac{i(1-R)}{2} e^{-K y}, & c<y<\infty,\end{cases}
\end{gathered}
$$

where $D_{1}, D_{2}, E_{1}, E_{2}$ are arbitrary constants. 
Now, for accommodating the origin and also the point at infinity, we have

$$
D_{1}=\frac{R}{2 K}, \quad E_{2}=0 .
$$

Let

$$
\int_{0}^{\infty} \xi A(\xi) \sin \xi y d \xi=g(y), \quad y \in G
$$

then using (3.11) and (3.12) in (3.10) and by Fourier sininversion,

$$
\begin{aligned}
\frac{\pi}{2} \xi A(\xi)= & \int_{G} g(y) \sin \xi t d t-\frac{i(1-R)}{2} \int_{\mathbf{B}} e^{-K t} \sin \xi t d t \\
& +E_{1} \int_{a}^{b} e^{K t} \sin \xi t d t .
\end{aligned}
$$

Substituting $A(\xi)$ from (3.13) into (3.9) and using the result $\int_{0}^{\infty}(\sin \xi y \sin \xi t / \xi) d \xi=$ $(1 / 2) \ln |(y+t) /(y-t)|$, we have

$$
\frac{1}{\pi} \int_{G} g(t) \ln \left|\frac{y+t}{y-t}\right| d t= \begin{cases}\frac{R}{2 K}\left(e^{K y}-e^{-K y}\right)+P_{1}(y), & 0<y<a, \\ D_{2} e^{K y}-\frac{R}{2 K} e^{-K y}+P_{1}(y), & b<y<c,\end{cases}
$$

where

$$
P_{1}(y)=\frac{1}{\pi}\left[-\frac{i(1-R)}{2} \int_{\mathbf{B}} e^{-K u} \ln \left|\frac{y+u}{y-u}\right| d u+E_{1} \int_{a}^{b} e^{K u} \ln \left|\frac{y+u}{y-u}\right| d u\right] .
$$

Now, (3.14) is identical with (1.1) with $f(y)$ as

$$
f(y)= \begin{cases}\frac{R}{2 K}\left(e^{K y}-e^{-K y}\right)+P_{1}(y), & 0<y<a, \\ D_{2} e^{K y}-\frac{R}{2 K} e^{-K y}+P_{1}(y), & b<y<c,\end{cases}
$$

where $P_{1}(y)$ is given by (3.15) and $R, D_{2}, E_{1}$ are unknown constants to be determined, and $G \equiv(0, a) \cup(b, c)$.

Now it is important to know the behaviour of $g(u)$ as $u \rightarrow a, b, c$. For this, let

$$
\phi_{x}(0, y)= \begin{cases}\omega(y), & y \in G \\ 0, & y \in \mathbf{B}(\text { by }(3.3))\end{cases}
$$

Using (3.7), we get

$$
\left(\frac{d}{d y}-K\right) \int_{0}^{\infty} \xi A(\xi) \sin \xi y d \xi=(1-R) i K e^{-K y}-\omega(y), \quad y \in G .
$$


Comparing it with (3.12), we get

$$
\omega(y)=-\frac{d g}{d y}+K g(y)+i K(1-R) e^{-K y} .
$$

Noting that (3.5) holds, we find that

$$
g(y) \sim O(|(y-t)|)^{1 / 2}, \quad \text { as } y \longrightarrow t, t=a, b, c .
$$

Thus $g$ satisfies the integral equation (2.1) with the condition (1.3), the solution of which is given by (2.32) together with the solvability conditions (2.31).

3.2. Determination of $R, D_{2}, E_{1}$. Knowing that $g(u)$ satisfies (1.3), we use $f(y)$ from (3.16) into (2.31) to obtain the following equations for unknown constants $R, D_{2}, E_{1}$ as

$$
\begin{aligned}
& a_{11} R+a_{12} D_{2}+a_{13} E_{1}=b_{1}, \\
& a_{21} R+a_{22} D_{2}+a_{23} E_{1}=b_{2}, \\
& a_{31} R+a_{32} D_{2}+a_{33} E_{1}=b_{3},
\end{aligned}
$$

where $a_{i j}$ s and $b_{j}$ s are given by

$$
\begin{aligned}
a_{11}= & \frac{1}{2}\left(A_{5}^{4}+\frac{K_{1}}{\Delta} \cdot A_{1}+\frac{K_{2}}{\Delta} \cdot A_{1}^{1}+H_{1}\right) \\
& +\frac{i}{2 \pi}\left(B_{1}^{4}\left(F_{1}\right)+\frac{K_{1}}{\Delta} \cdot B_{1}\left(F_{2}\right)+\frac{K_{2}}{\Delta} \cdot B_{1}^{1}\left(F_{2}\right)+\delta_{1}\left(F_{2}\right)+\delta_{3}\left(F_{2}\right)\right), \\
a_{12}= & -\left(\alpha_{3}^{4}(K, y)+\frac{K_{1}}{\Delta} \cdot \alpha_{3}(K, y)+\frac{K_{2}}{\Delta} \cdot \alpha_{3}^{1}(K, y)-\beta_{3}(K)\right), \\
a_{13}= & \gamma_{1}^{4}\left(F_{12}, y\right)-\gamma_{3}^{4}\left(F_{14}, y\right)+\frac{K_{1}}{\Delta} \cdot B_{1}\left(F_{22}\right)+\frac{K_{2}}{\Delta} \cdot B_{1}^{1}\left(F_{22}\right)+\delta_{1}\left(F_{22}\right)+\delta_{3}\left(F_{22}\right), \\
a_{21}= & \frac{1}{2}\left(A_{5}^{2}+i \cdot A_{2}^{2}\right), \quad a_{22}=-\alpha_{3}^{2}(K, y), \quad a_{2}^{2}(K, y), \\
a_{31}= & \left.\frac{1}{2}\left(A_{5}+i \cdot A_{2}\right), \quad a_{32}=-\alpha_{3}(K, y), y\right), \\
b_{1}= & \frac{i}{2 \pi}\left(B_{1}^{4}\left(F_{1}\right)+\frac{K_{1}}{\Delta} \cdot B_{1}\left(F_{2}\right)+\frac{K_{2}}{\Delta} \cdot B_{1}^{1}\left(F_{2}\right)+\delta_{1}\left(F_{2}\right)+\delta_{3}\left(F_{2}\right)\right), \\
b_{2}= & \frac{1}{2} A_{2}^{2}, \quad b_{3}=\frac{1}{2} A_{2},
\end{aligned}
$$


where

$$
\begin{gathered}
A_{1}^{n}=\alpha_{1}^{n}(K, y)-\alpha_{1}^{n}(-K, y)+\alpha_{3}^{n}(-K, y), \quad A_{1}=\alpha_{1}(K, y)-\alpha_{1}(-K, y)+\alpha_{3}(-K, y), \\
A_{2}^{n}=\alpha_{2}^{n}(-K, y)-\alpha_{4}^{n}(-K, y), \quad A_{2}=\alpha_{2}(-K, y)-\alpha_{4}(-K, y), \\
A_{5}^{n}=\alpha_{5}^{n}(-K, y)-\alpha_{3}^{n}(-K, y), \quad A_{5}=\alpha_{5}(-K, y)-\alpha_{3}(-K, y), \\
B_{1}^{n}(F)=\gamma_{1}^{n}(F, y)-\gamma_{3}^{n}(F, y), \quad B_{1}(F)=\gamma_{1}(F, y)-\gamma_{3}(F, y), \\
H_{1}=\beta_{1}(K, y)-\beta_{1}(-K, y)-\beta_{3}(-K, y), \\
F_{1}=F_{12}+F_{14}, \quad F_{12}=\int_{a}^{b} \frac{2 u e^{-K u}}{u^{2}-y^{2}} d u, \quad F_{14}=\int_{c}^{\infty} \frac{2 u e^{-K u}}{u^{2}-y^{2}} d u, \\
F_{22}=\int_{a}^{b} e^{-K u} \ln \left|\frac{y+u}{y-u}\right| d u, \quad F_{14}=\int_{c}^{\infty} e^{-K u} \ln \left|\frac{y+u}{y-u}\right| d u, \\
\alpha_{i}^{n}(K, y)=\int \frac{y^{n} e^{K y}}{R(y)} d y, \quad \alpha_{i}(K, y)=\int \frac{e^{K y}}{R(y)} d y, \\
\gamma_{i}^{n}(F, y)=\int \frac{y^{n}}{R(y)} \cdot F d y, \quad \gamma_{i}(F, y)=\int \frac{1}{R(y)} \cdot F d y, \\
\beta_{i}(K, y)=\int T^{*}(y) \cdot e^{K y} d y, \quad \delta_{i}(F, y)=\int T^{*}(y) . F d y,
\end{gathered}
$$

where, for integrals in (3.24), the range of integration is determined by the value of $i$ : for $i=1$, integration range is from 0 to $a$, for $i=2$, integration range is from $a$ to $b$, for $i=3$, integration range is from $b$ to $c$, for $i=4$, integration range is from $c$ to $\infty$, for $i=5$, integration range is from $-a$ to $a$.

Solving (3.21), the three unknowns can be determined and $g(u)$ can be known from (3.21). Hence from (3.12), $A(\xi)$ is known and $\phi$ can be obtained from (3.7). This solves the problem completely.

\section{Discussion}

The reflection coefficient $|R|$ has been numerically evaluated for various values of wave number $K b$ and presented graphically in Figures 4.1 and 4.2, respectively.

In Figure 4.1, we have taken $a / b=0.01$ and $c / b=1$. . It is observed that $|R|$ decreases at first, reaches a minimum, and then increases as $K b$ increases and ultimately becomes almost unity. This figure is in agreement with [1, Figure 1]. This type of behaviour is expected because for small value of ratio $a / b$, the upper end of the barrier is very near the free surface. Hence the short waves which are confined near the free surface are almost totally reflected by the barrier.

In Figure 4.2, we have taken $a / b=0.5$ and $c / b=2.0$. In this case, it is observed that $|R|$ decreases at first and becomes very small, and then again increases almost to unity as the wave number $K b$ increases. With further increase of $K b,|R|$ again decreases. This behaviour of $|R|$ for large wave number is expected because in absence of barrier near the 


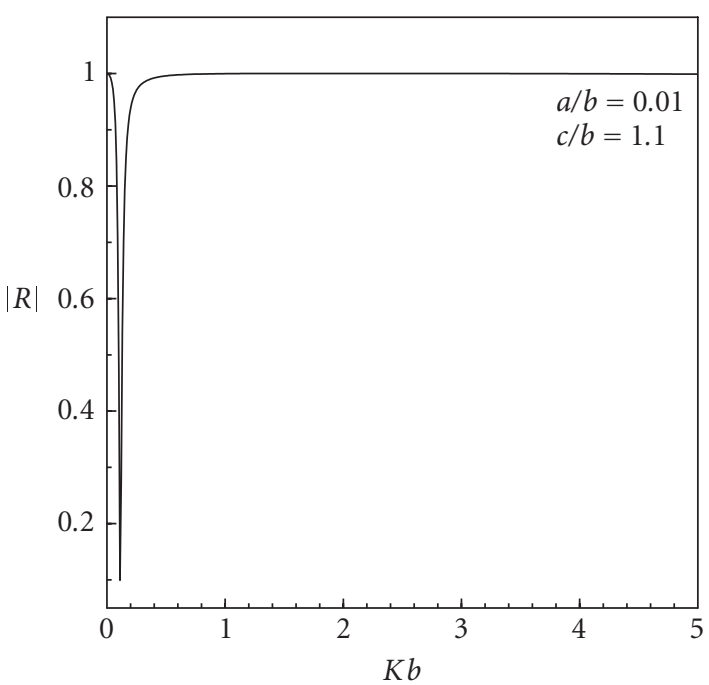

Figure 4.1

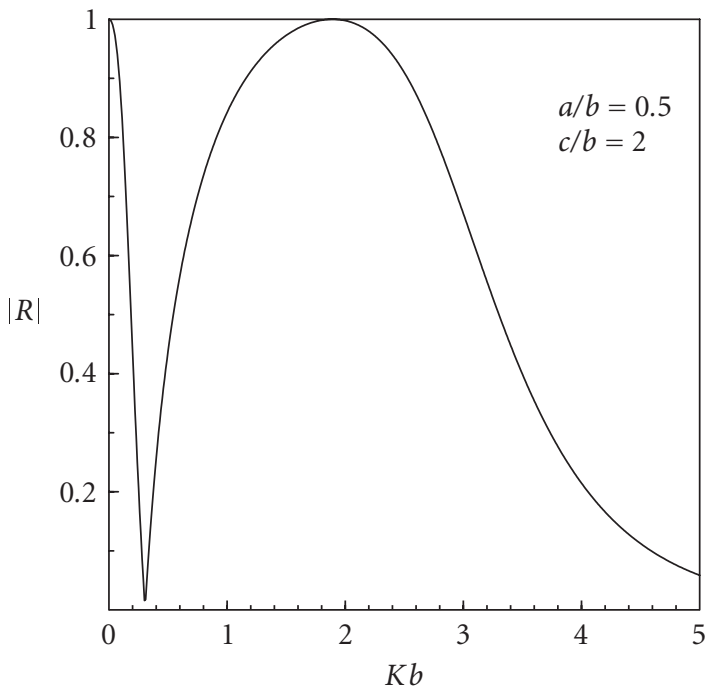

Figure 4.2

free surface, the short waves are totally transmitted. Also it is observed from Figure 4.2 that a gorge appears in the reflection coefficient for small values of wave number. This phenomenon occurs due to some resonance effect taking place owing to the interaction of flow with the gaps. Similar behaviour was also observed by Banerjea [1].

Thus in Figures 4.1 and 4.2, the behaviour of $|R|$ has been depicted for various values of wave number $K b$ and ratios $a / b$ and $c / b$. 
12 Weakly singular integral equation

\section{Conclusion}

We have used a function-theoretic method to obtain the solution of (1.1) and thereby utilize the boundedness property of the function satisfying (1.1), the problem of scattering of water waves by a submerged vertical barrier with a gap is solved easily.

\section{Acknowledgments}

Chiranjib Sarkar is grateful to CSIR for partial support to this work. This work is also partially supported by DST by a research project no. SR/S4/MS:263/05 through SB. Authors are thankful to Dr. Rupanwita Gayen for her help in numerical computation.

\section{References}

[1] S. Banerjea, Scattering of water waves by a vertical wall with gaps, Journal of Australian Mathematical Society. Series B 37 (1996), no. 4, 512-529.

[2] S. Banerjea and B. N. Mandal, Scattering of water waves by a submerged thin vertical wall with a gap, Journal of Australian Mathematical Society. Series B 39 (1998), no. 3, 318-331.

[3] A. Chakrabarti, S. R. Manam, and S. Banerjea, Scattering of surface water waves involving a vertical barrier with a gap, Journal of Engineering Mathematics 45 (2003), no. 2, 183-194.

[4] A. Chakrabarti and L. Vijaya Bharathi, Transmission of water waves through a gap in a submerged vertical barrier, Indian Journal of Pure and Applied Mathematics 22 (1991), no. 6, 491-512.

[5] S. R. Manam, A logarithmic singular integral equation over multiple intervals, Applied Mathematics Letters 16 (2003), no. 7, 1031-1037.

Sudeshna Banerjea: Department of Mathematics, Jadavpur University, Kolkata 700032, India

E-mail address: sbanerjee@math.jdvu.ac.in

Chiranjib Sarkar: Department of Mathematics, Jadavpur University, Kolkata 700032, India

E-mail address: chiranjib_s@hotmail.com 


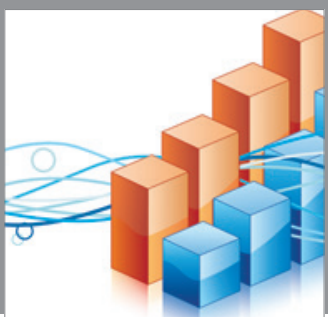

Advances in

Operations Research

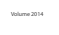

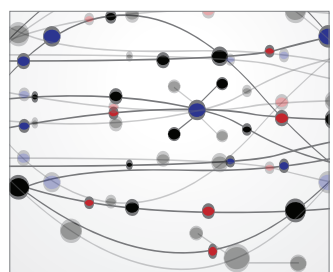

\section{The Scientific} World Journal
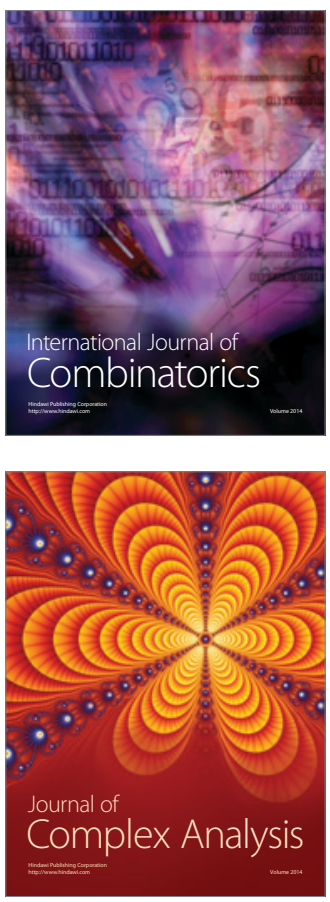

International Journal of

Mathematics and

Mathematical

Sciences
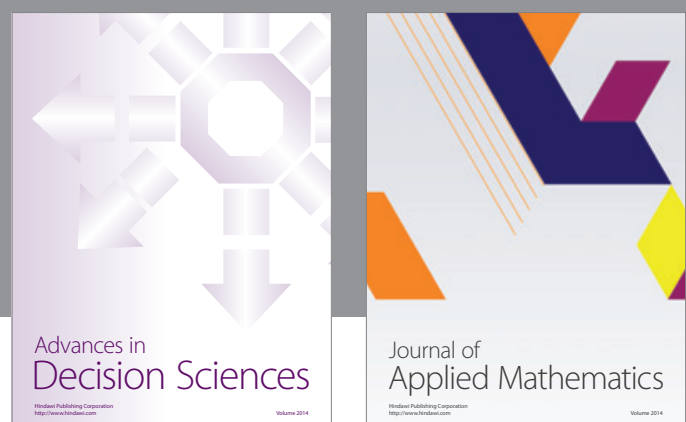

Journal of

Applied Mathematics
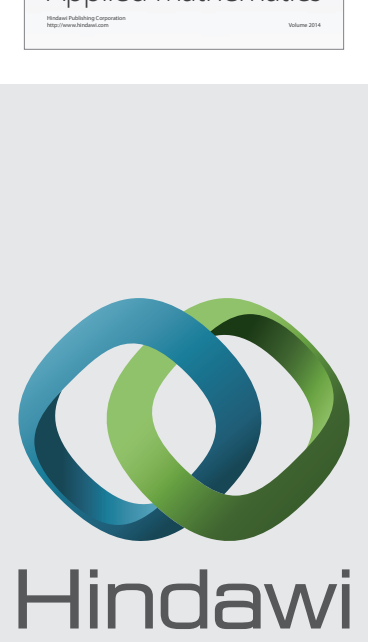

Submit your manuscripts at http://www.hindawi.com
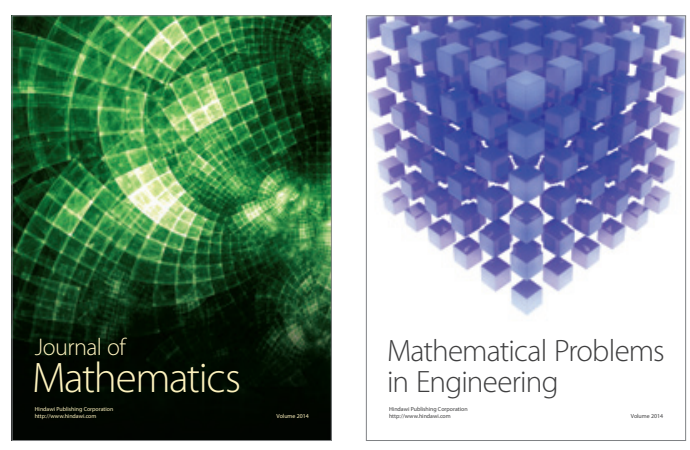

Mathematical Problems in Engineering
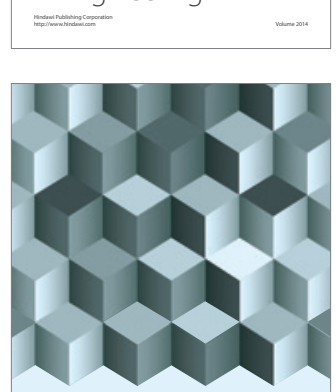

Journal of

Function Spaces
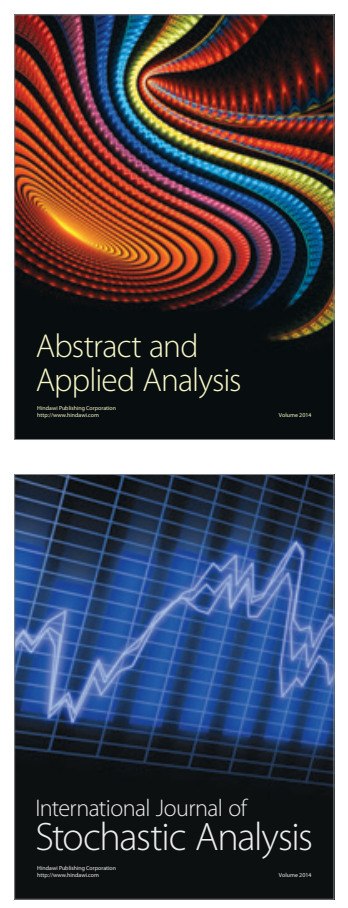

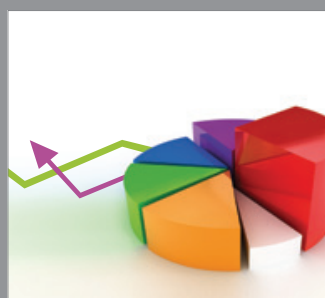

ournal of

Probability and Statistics

Promensencen
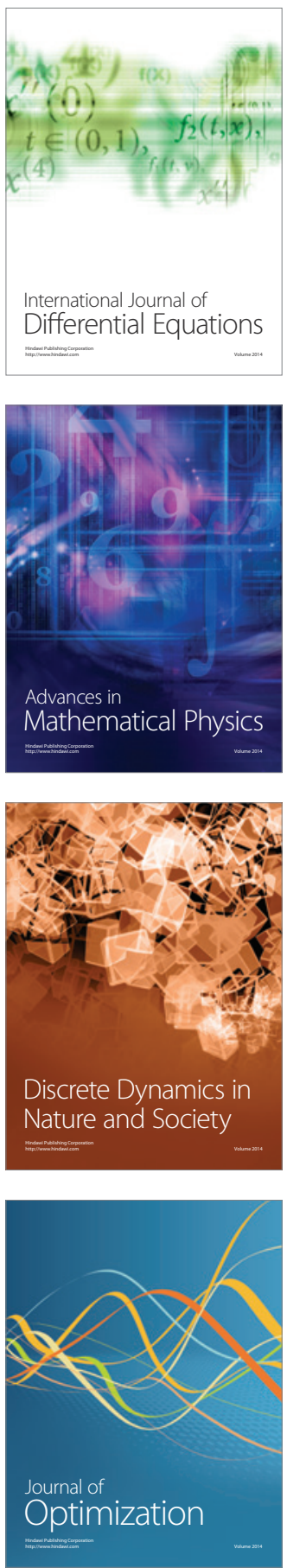